\title{
Flexible Ag Microparticle/MXene-Based Film for Energy Harvesting
}

Cite as

Nano-Micro Lett.

(2021) 13:201

Received: 28 July 2021

Accepted: 31 August 2021

Published online: 24 September 2021

(C) The Author(s) 2021

\author{
Yunpeng Jia ${ }^{1}$, Yamin Pan $^{1}$, Chunfeng Wang ${ }^{2,3}$, Chuntai Liu ${ }^{1}$, Changyu Shen ${ }^{1}$, \\ Caofeng $\mathrm{Pan}^{2}$, Zhanhu Guo ${ }^{4}$, Xianhu $\mathrm{Liu}^{1} \bowtie$
}

\section{HIGHLIGHTS}

- Flexible Ag microparticle/MXene-based energy-harvesting films were fabricated via simple spraying and hot-pressing.

- Optimal film shows high conductivity and effective electro/photo-thermal abilities.

- It can be easy assembled single-electrode TENG and the output voltage is enhanced by assembled two triboelectrification layers.

\begin{abstract}
Ultra-thin flexible films have attracted wide attention because of their excellent ductility and potential versatility. In particular, the energy-harvesting films (EHFs) have become a research hotspot because of the indispensability of power source in various devices. However, the design and fabrication of such films that can capture or transform different types of energy from environments for multiple usages remains a challenge. Herein, the multifunctional flexible EHFs with effective electro-/photo-thermal abilities are proposed by successive spraying Ag microparticles and MXene suspension between on waterborne polyurethane films, supplemented by a hot-pressing. The optimal coherent film exhibits a high electrical conductivity $\left(1.17 \times 10^{4} \mathrm{~S} \mathrm{~m}^{-1}\right)$, excellent Joule heating performance $\left(121.3{ }^{\circ} \mathrm{C}\right)$ at $2 \mathrm{~V}$, and outstanding photo-thermal performance $\left(66.2{ }^{\circ} \mathrm{C}\right.$ within $70 \mathrm{~s}$ under $\left.100 \mathrm{~mW} \mathrm{~cm}^{-1}\right)$.

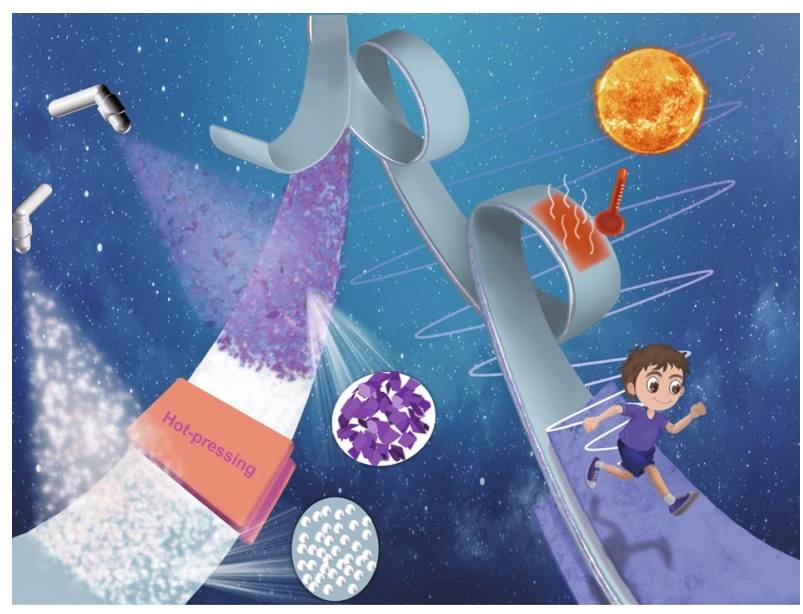
In addition, the EHFs-based single-electrode triboelectric nanogenerators (TENG) give short-circuit transferred charge of $38.9 \mathrm{nC}$, open circuit voltage of $114.7 \mathrm{~V}$, and short circuit current of $0.82 \mu \mathrm{A}$. More interestingly, the output voltage of TENG can be further increased via constructing the double triboelectrification layers. The comprehensive ability for harvesting various energies of the EHFs promises their potential to satisfy the corresponding requirements.
\end{abstract}

KEYWORDS Energy harvesting; MXene; Ag microparticle; Triboelectric nanogenerator

Yunpeng Jia and Yamin Pan contributed equally to this work.

$\bowtie$ Xianhu Liu, xianhu.liu@zzu.edu.cn

1 College of Materials Science and Engineering, Key Laboratory of Advanced Material Processing \& Mold (Ministry of Education), National Engineering Research Center for Advanced Polymer Processing Technology, Zhengzhou University, Zhengzhou 450002, People's Republic of China

2 National Center for Nanoscience and Technology (NCNST), Beijing Institute of Nanoenergy and Nanosystems, Chinese Academy of Sciences, Beijing 100083, People's Republic of China

3 College of Physics and Optoelectronic Engineering, Shenzhen University, Shenzhen 518060, People's Republic of China

4 Integrated Composites Laboratory, Department of Chemical \& Biomolecular Engineering, University of Tennessee, Knoxville, TN 37996 , USA 


\section{Introduction}

The consumption of natural resources such as fossil fuels continues to increase with the rapid development of the economy, and we are facing increasingly serious energy shortages and environmental damage. The efficient utilization of renewable resources (e.g., solar, wind, and tidal power) has been extensively explored; meanwhile, biomechanical energy from human movements is considered as an emerging way to provide the driving force for equipment [1-3]. In this context, the research of developing energy collection devices for ensuring energy security, realizing recycling, and promoting sustainable economic development is of great significance.

Energy-harvesting films (EHFs) are considered to be ideal candidate in this respect, which have been widely applied in solar cells [4-6], light-emitting components [7, 8], heating devices $[9,10]$, and so on. In general, the current EHFs collect various forms of energy by introducing fillers with different properties including carbon nanotubes, metal nanowires and conducting polymer to the elastic matrix [11-17]. Nevertheless, the nanofillers are easy to agglomerate during the preparation process and limit the application in practice $[18,19]$. Therefore, it is extremely important to select fillers with good dispersion and excellent performance. In particular, a newly emerged two-dimensional (2D) transition metal carbonitride noted as MXene, has shown surprising properties in the fields of electrical energy transmission and visible light absorption. The multilayer 2D material possesses high electrical conductivity $\left(\sim 10^{4} \mathrm{~S} \mathrm{~cm}^{-1}\right)$, as well as a large specific surface area and excellent dispersion, making it an ideal candidate for fabricating appreciable EHFs [20-23].

Triboelectric nanogenerator (TENG), converting mechanical energy, especially the human activities, into electricity by coupling the electrification and electrostatic induction, enables the energy storage and self-powered signal collection [24-26] and therefore has been highlighted combined with their portability, durability, material diversity for triboelectric layer (e.g., flexible polymers) and electrode (e.g., conductive hydrogel or fabric), and cost-effectiveness. The triboelectric layer usually has a strong triboelectrification effect and is less conductive or insulating, which can capture the transferred charges and retain them for an extended period of time, to build up the electrostatic charges and potential difference [27]. For example, polyurethane has been selected as a triboelectric layer more frequently due to its strong electronegativity, excellent flexibility, and environmental friendliness $[28,29]$. In another aspect, the electrode with a conductive network enables the electrons' transportation and the charging and discharging process. In particular, a hybrid electrode layer with the embedded conductive fillers can synergistically trap and block the charges, thereby further improving the electrical output capacity [30-33]. Through the constant contact-separation mode, TENG could convert mechanical energy into electricity by generating an objective electric current, enabling the construction of the self-powered systems.

Herein, the multifunctional EHFs were developed by spraying Ag microparticles (AgMPs) and MXene dispersed solution in sequence between on waterborne polyurethane (WPU) layers, featuring remarkable mechanical properties and high electrical conductivity. On the one hand, the EHFs can convert electrical energy and light energy into heat with a maximum of 121.3 and $66.2{ }^{\circ} \mathrm{C}$, respectively, indicating the reliability in application of electric-photo-thermal. On the other hand, electrons can be quickly transferred in the EHFs benefiting from the low resistance of the filler layer, and thus the composite films can be assembled into a single-electrode TENG (STENG) assisted with the strong triboelectric effect of the WPU layer. The EHFs with stable electrical output can provide power for electronic components, and their outstanding waterproof ability also broadens the application range. This work confirmed that the EHFs possess the feasibility of in-depth exploration in energy collection, utilization, and transformation.

\section{Experimental Section}

\subsection{Materials}

Lithium fluoride (LiF, 99\%) was purchased from Aladdin Reagent Co., Ltd. $\mathrm{Ti}_{3} \mathrm{AlC}_{2}$ powder (MAX, 400 mesh) was obtained from Jilin 11 Technology Co., Ltd. Ag microparticles (NO-M-004-3) with a size of $1 \mu \mathrm{m}$ were purchased from Shanghai Naiou Nano technology Co., Ltd., with a purity of $299.9 \%$. And commercial WPU was supplied by Guangzhou Dolphin New Materials Co., Ltd., with a concentration of $50 \mathrm{wt} \%$. Besides, Acrylic sheets and silicone rubber were purchased from Shanghai Yu Zhao Industrial Co., Ltd. 


\subsection{Synthesis of $\mathrm{Ti}_{3} \mathrm{C}_{2} \mathrm{~T}_{\mathrm{x}}$ MXene Nanosheets}

As previous research has stated, $\mathrm{Ti}_{3} \mathrm{C}_{2} \mathrm{~T}_{\mathrm{x}}$ MXene nanosheets were obtained by etching $\mathrm{Ti}_{3} \mathrm{AlC}_{2}$ in hydrofluoric acid (HF). Firstly, $\operatorname{LiF}(2 \mathrm{~g})$ was slowly introduced into a Teflon beaker containing $40 \mathrm{~mL} \mathrm{HCl}(9 \mathrm{M})$ with stirring continuously at $35{ }^{\circ} \mathrm{C}$ for $30 \mathrm{~min}$. Then, at an extremely slow pace, $2 \mathrm{~g}$ $\mathrm{Ti}_{3} \mathrm{AlC}_{2}$ was added to it. The etching process, which takes $24 \mathrm{~h}$ under magnetic agitation, aims to remove the aluminum layer. After it, the solution was centrifuged at $3500 \mathrm{rpm}$ for $10 \mathrm{~min}$ to collect the precipitate. The precipitate was further treated by ultrasound and centrifugation until reaching a suitable $\mathrm{pH}$ value (about 6). Then, the multilayered $\mathrm{Ti}_{3} \mathrm{C}_{2} \mathrm{~T}_{\mathrm{x}}$ MXene was dispersed in ethanol and ultrasonicated for $1 \mathrm{~h}$. After centrifugation at $10,000 \mathrm{rpm}$ for $10 \mathrm{~min}$, the obtained products were further treated by ultrasound to remove the unexfoliated $\mathrm{Ti}_{3} \mathrm{C}_{2} \mathrm{~T}_{\mathrm{x}}$ and collected. Finally, the $2 \mathrm{D} \mathrm{Ti}_{3} \mathrm{C}_{2} \mathrm{~T}_{\mathrm{x}}$ MXene nanosheets were obtained by freeze-drying at $-80^{\circ} \mathrm{C}$ for 3 days.

\subsection{Fabrication of EHFs}

Following the schematic exhibited in Fig. 1, the multilayered WPU/AgMPs/MXene/WPU films were fabricated by a layer-by-layer spraying process. Typically, different amounts of AgMPs or MXene were dispersed in an equal mixture of alcohol and water assisted by a hot plate to shorten the evaporation time during the spraying process. Then, the corresponding suspensions were evenly sprayed onto the Teflon substrate with a commercial spray gun at a distance of $10 \mathrm{~cm}$ and a pressure of 2 bar, respectively. Firstly, AgMPs are sprayed on the first layer of WPU, after it, the film is then transferred to a vacuum press $\left(100{ }^{\circ} \mathrm{C}, 1 \mathrm{MPa}\right)$ for $20 \mathrm{~min}$ to make the metal particles hot sintering process. Then, the MXene dispersion was coated on the sintered layer to form the composite films. Finally, the second layer of WPU is overlaid on it for coating. Ag layer, with different areal densities $\left(1,3\right.$, and $\left.5 \mathrm{mg} \mathrm{cm}^{-2}\right)$, was used as the core layer along with the MXene layer.

\subsection{Fabrication of Sandwich-Structured STENG}

The preparation process of STENG is simple and efficient. Before spraying the MXene layer, a copper wire was introduced, and then the resulting composite film was affixed to the acrylic substrate with double-sided tape. What needs to be explained is that the top WPU layer acts as a positive dielectric material and the MXene and Ag layer act as a synergistic electrode.

\subsection{Characterization}

The morphology of the MxAgy films was characterized by field emission scanning electron microscopic (FE-SEM, JSM-7001F) at an accelerating voltage of $20 \mathrm{kV}$. The volume conductivity of samples was performed with the aid of a digital multimeter (Tektronix DMM4050), and the value can be calculated by the following equation: $\sigma=L / \mathrm{RS}$, where $\sigma$ is the volume conductivity, $L$ is the length of both ends, $R$ is the volume resistance, and $S$ is the cross section area of the film. The transmittance spectrum was measured by UV-Vis NIR absorption spectrometer (Cary 5000) with the measured wavelength range of 300-2400 nm.

A universal test platform (UTM2203, Shenzhen Suns Technology Stock Co., Ltd., China) controlled by a custom computer was used and equipped with a $100 \mathrm{~N}$ force sensor to measure mechanical properties. The stress-strain curves of MxAgy films were carried out under uniaxial test conditions with a crosshead speed of $5 \mathrm{~mm} \mathrm{~min}^{-1}$.

The electro-photo-thermal property was characterized by an infrared (IR) thermal imaging instrument (FLIR, E60), and the temperature change was real-time-recorded. The difference is that the electro-thermal test was done by applying a voltage to the sample, while the photo-thermal test was done by exposing the membrane to a solar simulator. The value of $\mathrm{Q}_{\mathrm{SC}}, \mathrm{V}_{\mathrm{OC}}$, and $\mathrm{I}_{\mathrm{SC}}$ was precisely recorded by an electrometer (Keithley-6514). It was carried out under the frequency-controlled force generated by the motor.

\section{Results and Discussion}

\subsection{Fabrication and Structure of the MxAgy EHFs}

The schematic process of multifunctional EHFs is illustrated in Fig. 1a. Briefly, on one side of the prepared WPU film, AgMPs are sprayed and then subjected to a certain condition of hot-pressing treatment. The purpose of the hot-pressing process is mainly for sintering AgMPs. The activation energy required for surface diffusion is relatively low, and the high temperature can just provide the 

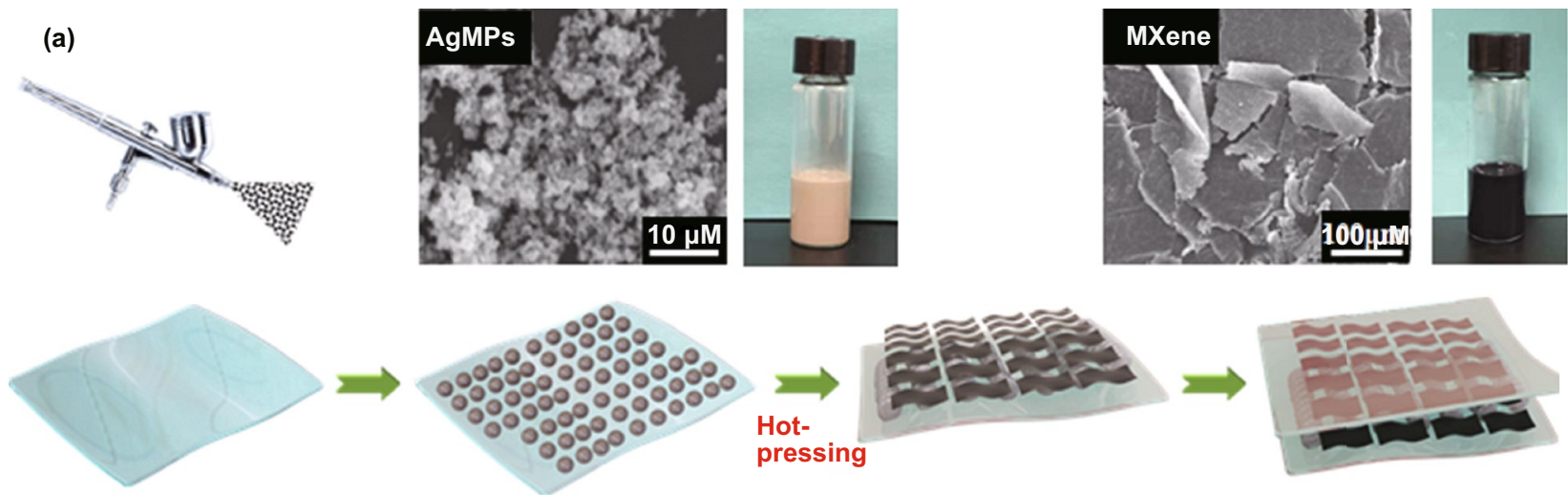

(b)
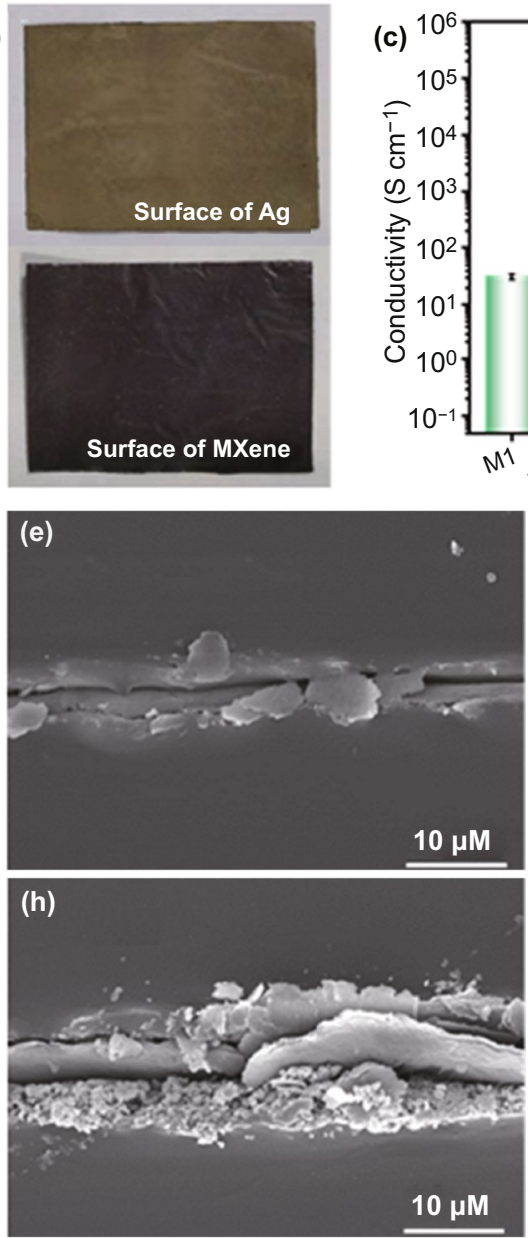
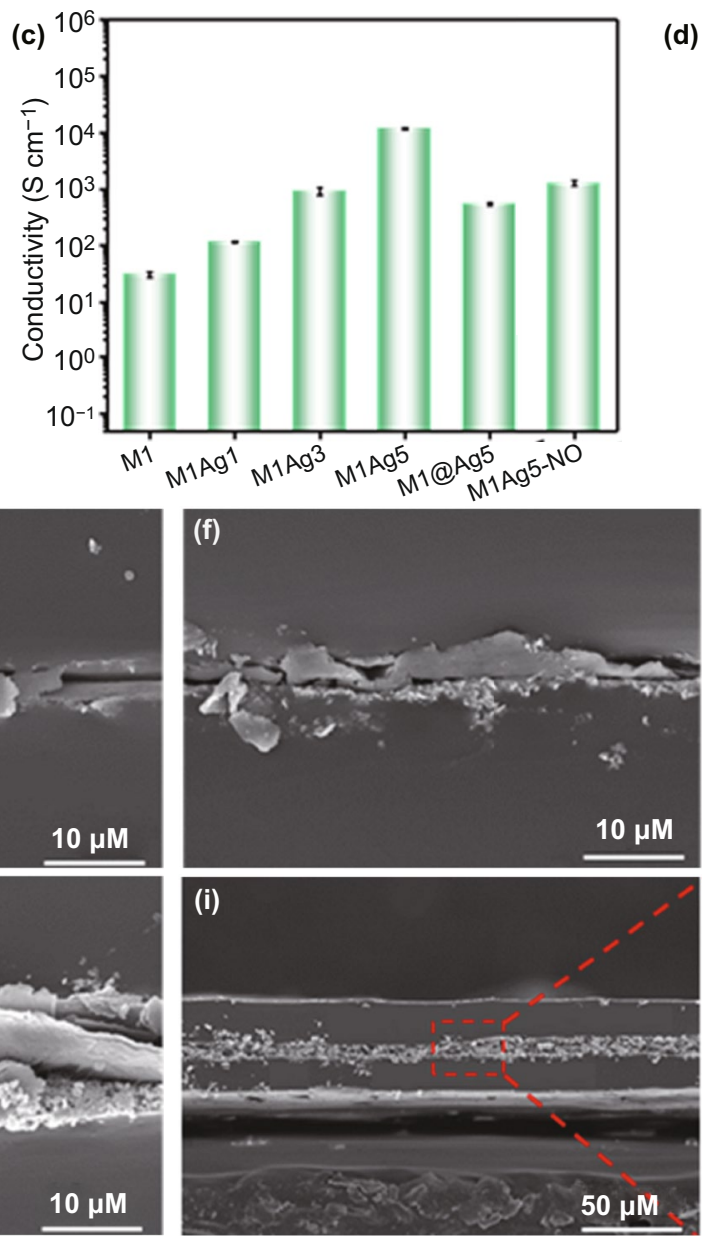

(d)
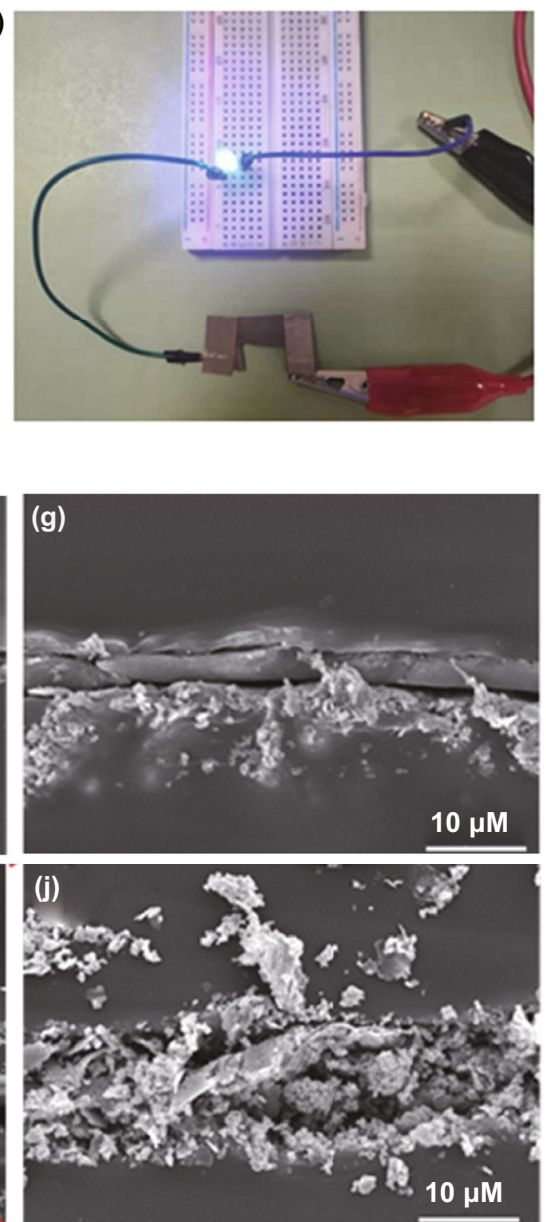

Fig. 1 a Preparation process of the EHFs. The illustrations are SEM images of AgMPs and MXene sheets as well as their dispersed solutions. b Digital photographs of both sides of EHFs. c Electrical conductivity of EHFs. d Digital photographs of lighting up LED by a power supply. Cross-sectional SEM images of e M1, f M1Ag1, g M1Ag3, h M1Ag5 and i-j M1@ Ag5 EHFs

required energy. Furthermore, the surface diffusion has a certain effect on the formation and expansion of the sintering neck, and the necks between the particles become wider under high-pressure conditions to form an effective metallic bonding [34, 35]. As shown in Fig. S1, before sintering, the conductivity of the Ag layer is relatively low, which is due to a sparse percolation network of particles. During the hotpressing process, AgMPs are transformed into a coherent 
microstructure at an elevated temperature, and subsequently, the ligament size of nano-porous Ag increases, leading to the complete bonding of AgMPs with each other (Fig. S2). After hot-pressing, MXene dispersion and WPU emulsion were sprayed sequentially. The corresponding composite films are described as MxAgy, where $\mathrm{x}$ and $\mathrm{y}$ represent the areal density $\left(\mathrm{mg} \mathrm{cm}^{-2}\right)$ of MXene and AgMPs, respectively. In addition, a mixed MXene and AgMPs was prepared, which is named M1@ $\mathrm{Ag} 5$ for comparison. M1 Ag5-NO represents the sample without the hot-pressing treatment of AgMPs. The two sides of EHFs have different colors (Fig. 1b), and the electrical conductivity of EHFs increased as the AgMPs contents increased (Fig. 1c), enabling the lighting-up of a LED under a power supply (Fig. 1d).

The scanning electron microscopy (SEM) images of films show a typical layer-by-layer structure (Fig. 1e-j). In Fig. 1e, it can be clearly seen that MXene sheets are sandwiched between WPU layers. With the introduction of AgMPs, an obvious granular layer appeared below MXene. It is worth noting that due to the effect of the hot-pressing, some AgMPs will be pressed into the bottom WPU layer, which may enhance the mechanical properties of the film for the embedding of fillers (Fig. S3). More importantly, no obvious interface defects are discovered between these layers, which is conducive to ensure performance stability in different conditions. In contrast to the mixed-phase film of M1@Ag5, AgMPs are randomly distributed on the MXene layer, and the overall structure was relatively loose. Moreover, the thin thickness of MxAgy $(\sim 50 \mu \mathrm{m})$ and conductive layer $(\sim 10 \mu \mathrm{m})$ make it great potential in miniaturized and lightweight regions.

\subsection{Electric-Thermal and Photo-Thermal Performances}

According to the principle of Joule heating, heat can be generated when electric currents pass through a conductor. Joule's law can be expressed by Eq. 1:

$Q=U I t=I^{2} R t=\frac{U^{2}}{R} t$

where $Q, U, I, R$, and $t$ represent generated Joule, applied voltage, current, resistance, and operating time, respectively $[36,37]$. For the convenience of research, all the tests are characterized in the surface of AgMPs. Figure $2 a-c$ reflects the timely feedback of Joule-heating temperature of M1, $\mathrm{M} 1 \mathrm{Ag} 1, \mathrm{M} 1 \mathrm{Ag} 5$ under different voltages. The temperature of M1Ag1 and M1 Ag5 can rapidly heat up to the platform of 72.1 and $121.3{ }^{\circ} \mathrm{C}$ within $20 \mathrm{~s}$, with applied voltages of 4 and $2 \mathrm{~V}$, respectively. However, the control group M1 can only heat up to about $43.6{ }^{\circ} \mathrm{C}$, even at a high voltage of $14 \mathrm{~V}$, ascribing to the low conductivity of M1, as depicted in Fig. 1c. The M1 Ag5 can maintain a stable temperature platform under the long period of working conditions (Fig. S4). By applying different gradients of external voltage, the EHFs exhibit controllable cycle stability and temperature responsiveness (Fig. 2d). During the energization process, the temperature distribution on the film surface is uniform, and the brightness of the infrared image obviously increases as the voltage rises. Moreover, the stability of the heater is also an important indicator of whether it can work for a long term. As shown in Fig. 2e, the voltage-current curves of all three samples are described, indicating that the constant resistance of the composite films and the height conforms to Ohm's law through the fitting curves under different voltages. While, due to the polymer matrix, the heating upper limit of the heater is subject to certain restrictions.

As shown in Fig. 2f, contrast to the pure WPU film, the EHFs of M1, Ag5, and M1Ag5 exhibit excellent photothermal abilities, which can be heated to 54.2, 64.3, and $66.2{ }^{\circ} \mathrm{C}$, respectively, within $70 \mathrm{~s}$ when exposed to 1 sun irradiation $\left(100 \mathrm{~mW} \mathrm{~cm}^{-2}\right)$, ascribing to the effective photothermal effect of AgMPs and MXene. The higher overall temperature of the MxAgy films compared to M1 and Ag5 confirms the synergetic photo-thermal effect of MXene and AgMPs (Fig. 2g, a clearer photograph is shown in Fig. S5), for the coherent oscillation of the surface electrons induced and localized surface plasmon resonance (LSPR) of metal particles by light [38, 39]. Similarly, the semi-metallic properties of MXene also exhibit this similar property [40, 41]. When AgMPs of $1 \mathrm{mg} \mathrm{cm}^{-2}$ are sprayed, the photo-thermal effect of the EHFs is hardly improved. What's more, it can be concluded that the hybrid EHFs possess a higher absorbance in the visible wavelength range of 400 to $800 \mathrm{~nm}$ (Fig. S6), which provides the most important factor for the enhancement of the photo-thermal effect. Figure $2 \mathrm{~h} \mathrm{dem}$ onstrates that the photo-thermal performance of the film is stable under the cyclic irradiation of $100 \mathrm{~mW} \mathrm{~cm}^{-2}$, and the photo-to-heat output capacity does not decrease obviously. Furthermore, the temperature of the $\mathrm{M} 1 \mathrm{Ag} 5$ film rises from 42.3 to $76.5^{\circ} \mathrm{C}$ with the increase in light irradiation intensity from 50 to $200 \mathrm{~mW} \mathrm{~cm}^{-2}$, and the infrared image on the right visually reflects this difference (Fig. 2i). It is worth noting that the photo-thermal output of the film does not show a linear relationship with the illumination intensity 

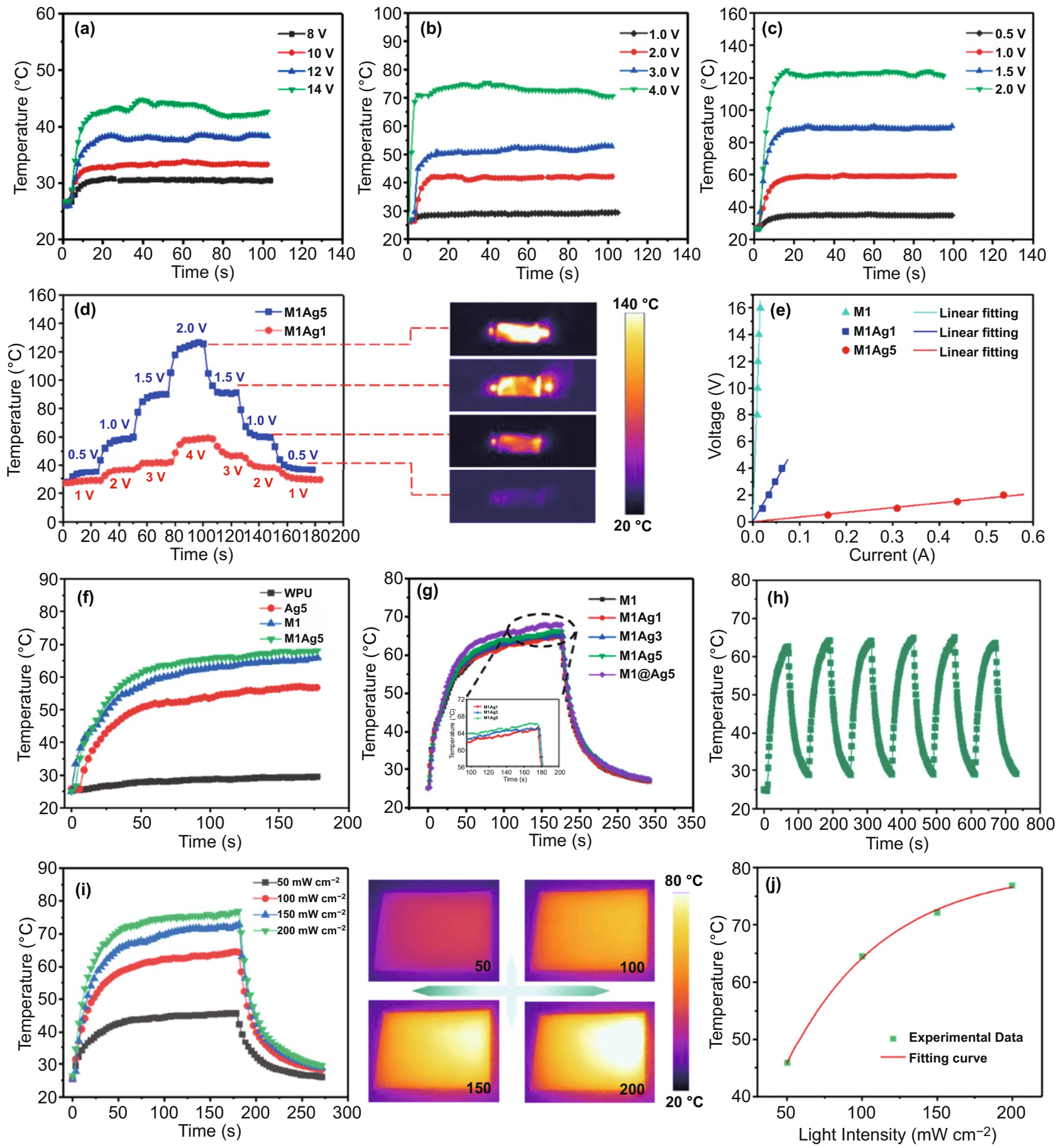

Fig. 2 Temperature variation at different applied voltages of a M1, b M1Ag1, c M1Ag5 EHFs. d Temperature evolution of M1Ag1 and M1Ag5 upon gradient changed voltages. e Voltage-current curves. f, $\mathbf{g}$ Temperature of different films under $100 \mathrm{~mW} \mathrm{~cm}^{-2}$. $\mathbf{h}$ Temperature variation curve under cycles of on-off light illumination. i Temperature difference of M1A5 film under different irradiated power densities. $\mathbf{j}$ Experimental data and fitting curve of peak temperature versus the irradiated power density

(Fig. 2j), which may be caused by the inherent property of the high thermal conductivity of the AgMPs. Figure S7 reveals that with the increase in AgMPs content, the thermal conductivity in the vertical direction of the MxAgy EHFs gradually increases. 


\subsection{Electrical Output Performance of the EHFs-based STENG}

In addition to the excellent flexibility of WPU [42], WPU exhibits a remarkable ability to gain or lose electrons when in contact with different materials as well, allowing it to be the triboelectric layer in STENG [43]. In this work, the STENG can be prepared easily by introducing a copper wire before spraying MXene (Fig. 3a). Figure 3b systematically illustrates the structure and working principle of the STENG. At first, the silicone rubber and WPU layer is determined as the negative and positive triboelectric materials, respectively, due to their difference in relative polarity
(I). When the two subjects contact, negative charges will produce on silicon rubber due to its strong negative charge capturing ability, and the same amount of positive charge is generated on the surface of the WPU layer to keep the potential in balance (II). When the silicone rubber separates from the WPU layer, the unscreened positive charges will induce the accumulation of negative charges in the AgMPs/MXene interface. Meanwhile, the instantaneous electrons will flow from the ground to the AgMPs/MXene layer (III). When the separation degree between the rubber and the WPU layer reaches the maximum, the potential in the STENG system will reach equilibrium and the flow of free electrons will also terminate (IV). As rubber and WPU approach again, (a)

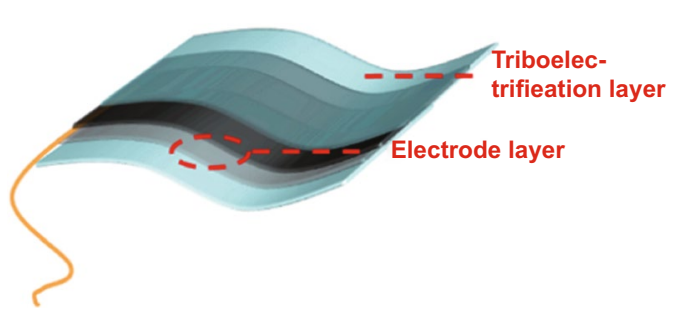

(c)
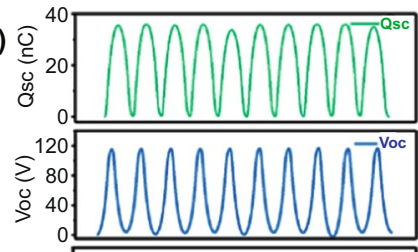

经 0.50

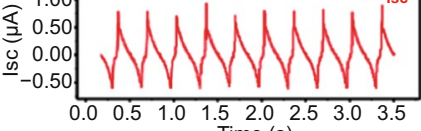

$\begin{array}{ccccc}0.0 & 0.5 & 1.0 & 1.5 & 2.0 \\ & & & & \\ \text { Time (s) }\end{array}$

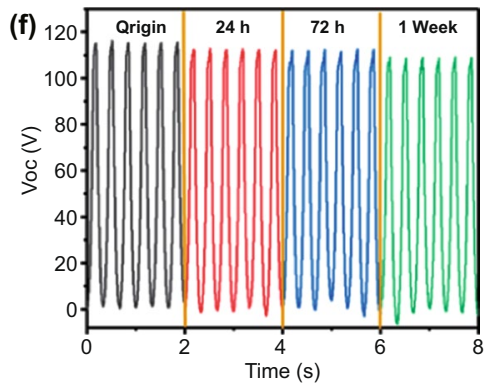

(g) (b)

(d)

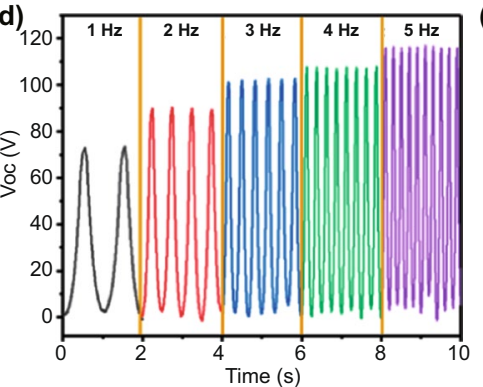

MXene
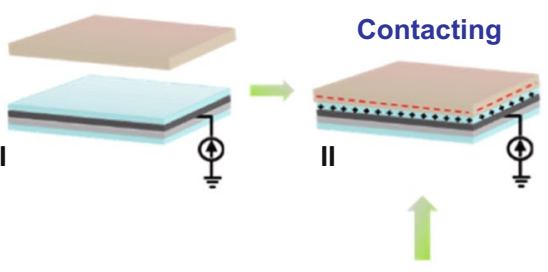

Pressing
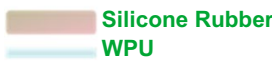
Ag Particle

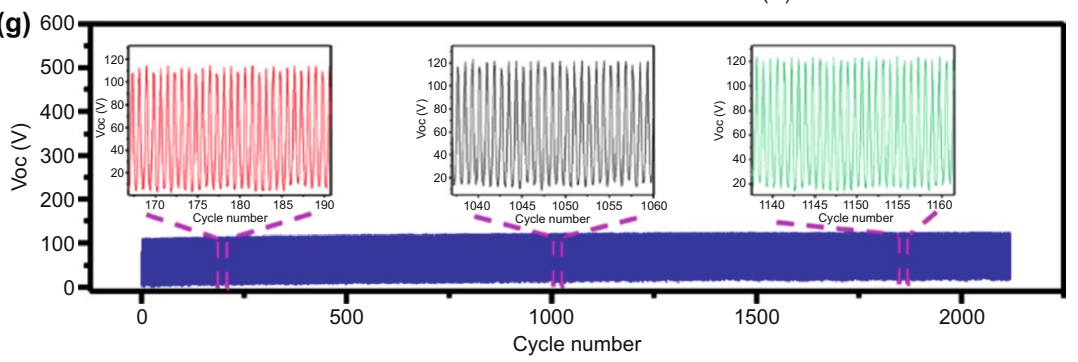

Fig. 3 a EHFs-STENG and $\mathbf{b}$ its working mechanism diagram. $\mathbf{c}$ The corresponding $\mathrm{V}_{\mathrm{OC}}, \mathrm{I}_{\mathrm{SC}}$, and $\mathrm{Q}_{\mathrm{SC}}$ of the STENG. $\mathbf{d} \mathrm{V}_{\mathrm{OC}}$ of the STENG with various frequencies (fixed pressure $10 \mathrm{~N}$ ). e The output performance under the resistance of $1 \mathrm{k} \Omega-1 \mathrm{G} \Omega$. $\mathbf{f} \mathrm{V}_{\mathrm{OC}}$ of STENG in a wet environment within one week. $\mathbf{g}$ Long-term stability test of the STENG 
the electrons will flow from the MxAgy conductive layer to the ground, indicating a reversed operation mechanism (V). By repeating the contact-separation procedure mentioned above, an alternating electrical signal can be generated. The changes of the induced voltage of rubber and WPU layer during the movement process can be intuitively illustrated through the simulation of COMSOL (Fig. S8).

The electrical output performance of STENG is affected by different factors such as pressure, frequency, and contact material [44-48]. Therefore, a linear motor is used to study the property of EHFs-based STENG in the periodic contactseparation motions. The contact area is chosen as $40 \times 40 \mathrm{~mm}^{2}$ and the tapping force is $10 \mathrm{~N}$ (frequency: $5 \mathrm{~Hz}$ ). As shown in Fig. 3c, the transferred short-circuit charge $\left(\mathrm{Q}_{\mathrm{SC}}\right)$, the opencircuit voltage $\left(\mathrm{V}_{\mathrm{OC}}\right)$ and the short-circuit current $\left(\mathrm{I}_{\mathrm{SC}}\right)$ of the STENG are $38.9 \mathrm{nC}, 114.7 \mathrm{~V}$ and $0.82 \mu \mathrm{A}$, respectively. The variation of output performance dependence on different AgMPs content is shown in Fig. S9. Increasing output $\mathrm{V}_{\mathrm{OC}}$ and $\mathrm{I}_{\mathrm{SC}}$ performance of STENG is due to the following reasons: the outstanding electrical conductivity of AgMPs causes the decrease in surface resistance significantly; the high AgMPs content leads to more effective contact area with the WPU layer. The $\mathrm{V}_{\mathrm{OC}}$ of STENG at different frequencies is also studied (Fig. 3d). The $\mathrm{V}_{\mathrm{OC}}$ increases accordingly with the motor frequency, which is mainly because the higher the frequency, the smaller the neutralization of positive and negative charges in the dielectric layer and the triboelectrification layer during contact, resulting in more charges gathering on the electrode and high electrical output [45]. In addition, the influence of tapping force on STENG electrical output capacity can be seen in Fig. S10. With the augment of applying pressure, the effective triboelectric area increases due to the deformation of the polymer materials [27, 49]. Figure 3e displays the output voltage, current density, and power density of the STENG by connecting various external resistors of 1 $\mathrm{k} \Omega$ to $1 \mathrm{G} \Omega$. As shown in the chart, following Ohm's law, the voltage of the external resistance rises while the current decreases with the resistance increasing. The power density $(P)$ can be calculated by Eq. 2 :

$P=\frac{U^{2}}{R A}=\frac{U I}{A}$

where $U$ and $I$ represent the output voltage and current of the STENG, respectively. $A$ is the contact area, $R$ stands for the magnitude of the resistance. When the external load resistance is about $250 \mathrm{M} \Omega$, the output power density of EHFs-based STENG can reach a maximum of approximately $186.8 \mathrm{~mW} \mathrm{~m}^{-2}$. The repeatability of electrical output is crucial for STENG equipment, herein, the STENG still maintains satisfactory stable outputs after over 2000 pressingreleasing circulations (Fig. 3g), showing the reliability of the device in practical application. Besides, the waterproof performance of STENG was characterized. Surprisingly, the $\mathrm{V}_{\mathrm{OC}}$ remained at a similar level even after soaking in deionized water for 1 week due to the excellent waterproof performance of WPU (Fig. 3f). Additionally, the EHFs-based STENG also has a good responsiveness to human skin. As shown in Fig. S10 and Movie S1, the STENG can generate identifiable voltage signals by finger tapping and regular steps with different frequencies. This work provides a promising exploration of EHFs-based STENG in the field of human body sensors.

A self-powered system is assembled to verify the feasibility of EHFs-based STENG in practical application. As depicted in Fig. 4a, the STENG was linked to capacitor, LEDs, and electronic watch through a rectified circuit that converts the output alternating current to direct current. The charging capability of the STENG was measured by charging a $0.22 \mu \mathrm{F}$ commercial capacitor with different operating frequencies $(1-5 \mathrm{~Hz})$ and capacitors of various capacity sizes $(0.22-10 \mu \mathrm{F})$ at a frequency of $5 \mathrm{~Hz}$ (Fig. 4b, c). It exhibits that the charging rate increases significantly with the increasing motor frequency, which is mainly attributed to the higher contact-separation frequency of the triboelectrically layer, leading to the more accumulation of the friction charge. And, as the capacitance increases, the charging rate of the capacitor slows down reasonably. Moreover, the STENG can impel more than 20 commercial LEDs to light up by periodically clapping hands at a certain frequency (Fig. $4 \mathrm{~d}$ and Movie S2). Further, combining with an energy storage unit, the STENG can provide driving power for portable electronic devices, such as electronic watches (Fig. 4e), electronic thermometers (Fig. 4f), etc. These results confirm that the EHFs-based STENG has the potential to be further explored in self-charging power supply system [50]. Particularly, the structure of the double triboelectrification layers is constructed to greatly increase the output voltage of STENG (Fig. 4g), which provides a potential direction for future research. The possible mechanism is shown in Fig. S12. The polarization and accumulation of charge 


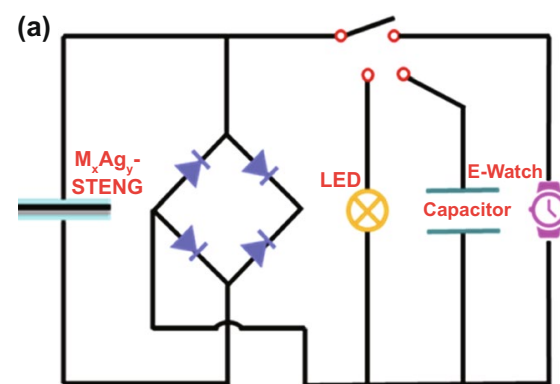

(d)

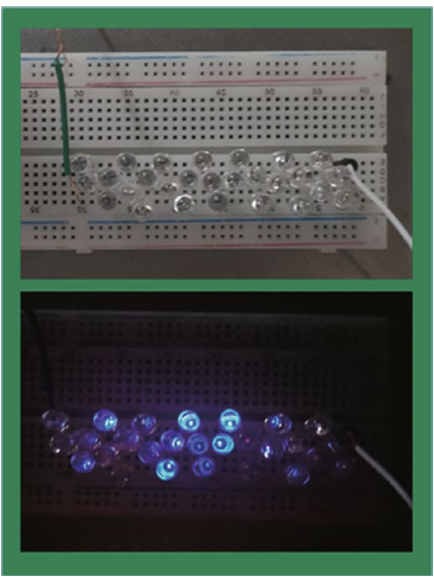

(g)
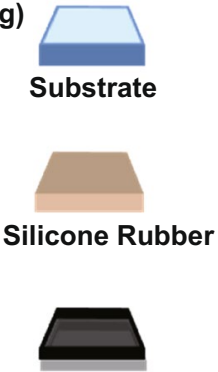

EHFs-STENG

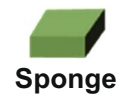

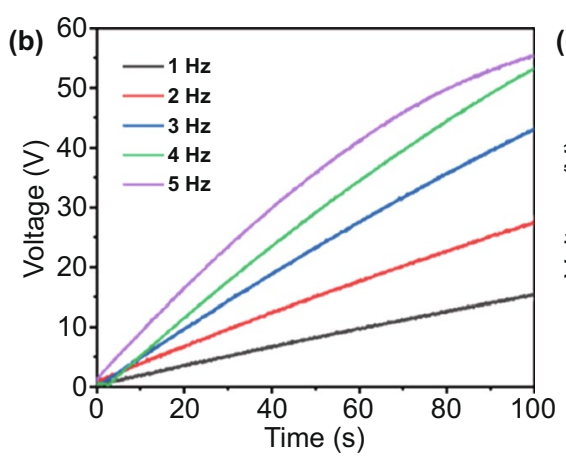

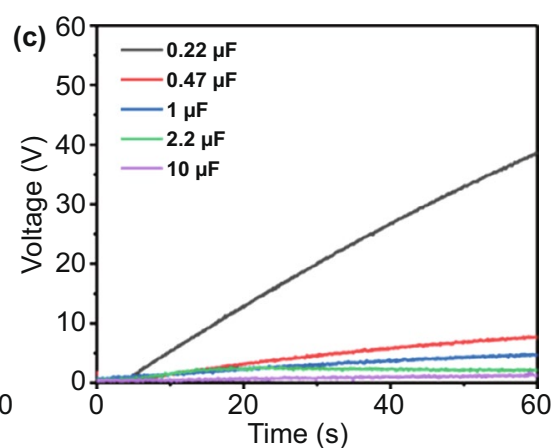

(e)

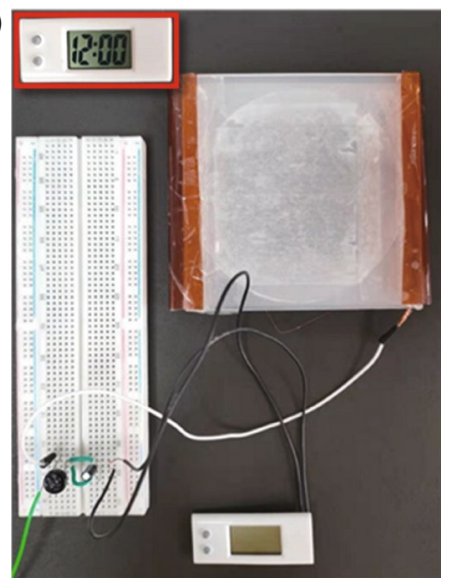

(f)

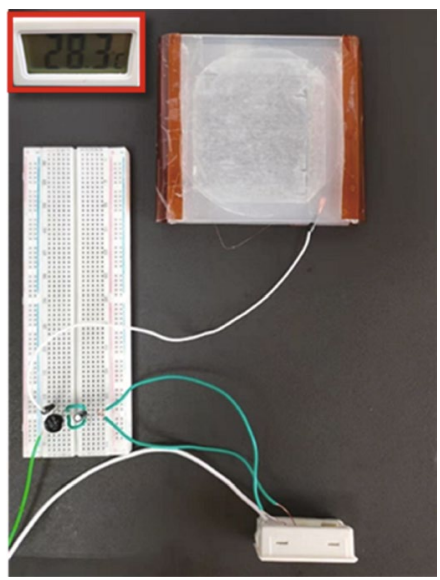

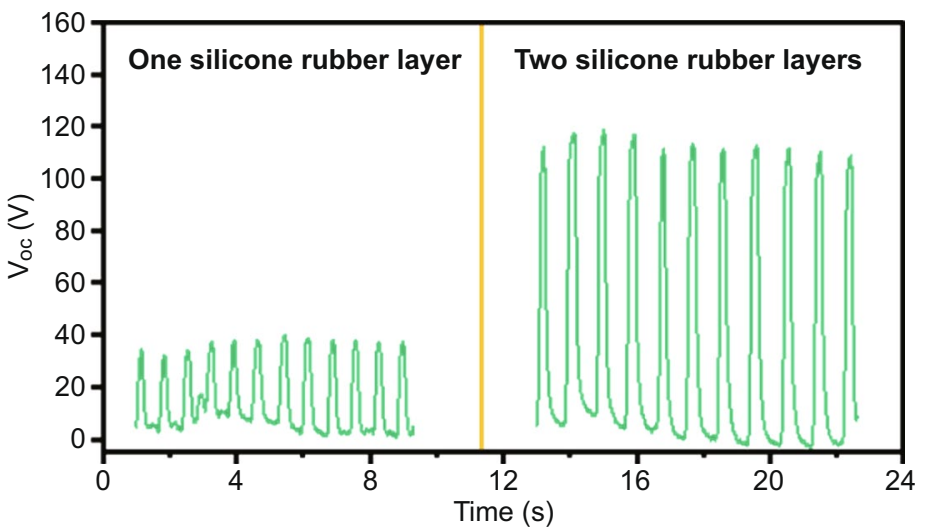

Fig. 4 a Self-charging power supply system based on the equivalent circuit diagram. The charging ability of the EHFs-based STENG b with various frequencies $(1-5 \mathrm{~Hz})$ for charging a $0.22 \mu \mathrm{F}$ commercial capacitor and c charging different capacitors $(0.22-10 \mu \mathrm{F})$ at a frequency of $5 \mathrm{~Hz}$. The electricity output from the STENG can drive $\mathbf{d}$ LEDs to shine, e electronic watches to run, and $\mathbf{f}$ electronic thermometers to work. $\mathbf{g}$ $\mathrm{V}_{\mathrm{OC}}$ of the EHFs-based STENG under single and double triboelectrification layers

occurred simultaneously in the upper and lower layers of WPU, and the design of double triboelectrification layers shorts the distance and speeds up the contact efficiency. The larger contact area, the higher contact-separation frequency, and the more significant the potential difference formed.

\section{Conclusions}

In summary, EHFs were fabricated via the technology of layer-by-layer spraying and hot-pressing. The cross-section SEM images reveal the effective establishment of a stratified structure in EHFs. As expected, the high electrical 
conductivity and absorbance of the network constructed by the fillers endow the EHFs with low-voltage-driven Joule heating effect and remarkable photo-thermal property, respectively. Furthermore, due to the WPU layer is easy to gain or lose electrons when contacting different polarities materials, the EHFs-based STENG is verified to be able to effectively convert mechanical energy into electrical energy output and provide power for microelectronic devices in continuous contact-separation mode. This work illustrates the great potential applications of EHFs for energy harvesting to achieve better energy recycling.

Acknowledgements The authors thank National Natural Science Foundation of China (51803190) and National Key R\&D Program of China (2019YFA0706802) for financial support.

Open Access This article is licensed under a Creative Commons Attribution 4.0 International License, which permits use, sharing, adaptation, distribution and reproduction in any medium or format, as long as you give appropriate credit to the original author(s) and the source, provide a link to the Creative Commons licence, and indicate if changes were made. The images or other third party material in this article are included in the article's Creative Commons licence, unless indicated otherwise in a credit line to the material. If material is not included in the article's Creative Commons licence and your intended use is not permitted by statutory regulation or exceeds the permitted use, you will need to obtain permission directly from the copyright holder. To view a copy of this licence, visit http://creativecommons.org/licenses/by/4.0/.

Supplementary Information The online version contains supplementary material available at https://doi.org/10.1007/ s40820-021-00729-w.

\section{References}

1. M. Salauddin, R.M. Toyabur, P. Maharjan, M.S. Rasel, H. Cho et al., Design and experimental analysis of a low-frequency resonant hybridized nanogenerator with a wide bandwidth and high output power density. Nano Energy 66, 104122 (2019). https://doi.org/10.1016/j.nanoen.2019.104122

2. M. Salauddin, S.M.S. Rana, M. Sharifuzzaman, M.T. Rahman, C. Park et al., A novel MXene/ecoflex nanocomposite-coated fabric as a highly negative and stable friction layer for highoutput triboelectric nanogenerators. Adv. Energy Mater. 11, 2002832 (2021). https://doi.org/10.1002/aenm.202002832

3. K. Qin, C. Chen, X. Pu, Q. Tang, W. He et al., Magnetic array assisted triboelectric nanogenerator sensor for real-time gesture interaction. Nano-Micro Lett. 13, 51 (2021). https://doi. org/10.1007/s40820-020-00575-2

4. C. Li, Y. Pan, J. Hu, S. Qiu, C. Zhang et al., (2020) Vertically aligned $2 \mathrm{D} / 3 \mathrm{D} \mathrm{Pb}-\mathrm{Sn}$ perovskites with enhanced charge extraction and suppressed phase segregation for efficient printable solar cells. ACS Energy Lett. 5, 1386-1395. https:// doi.org/10.1021/acsenergylett.0c00634

5. C. Wang, J. Hu, C. Li, S. Qiu, X. Liu et al., Spiro-linked molecular hole-transport materials for highly efficient inverted perovskite solar cells. Solar RRL 4, 1900389 (2020). https:// doi.org/10.1002/solr.201900389

6. C. Chen, J. Hu, Z. Xu, Z. Wang, Y. Wang et al., Natural methionine-passivated $\mathrm{MAPbI}_{3}$ perovskite films for efficient and stable solar devices. Adv. Compos. Hybrid Mater. (2021). https://doi.org/10.1007/s42114-021-00238-9

7. J. Zhuang, J. Sun, D. Wu, Y. Liu, R.R. Patil et al., Multifactor analysis on thermal conductive property of metal-polymer composite microstructure heat exchanger. Adv. Compos. Hybrid Mater. 4, 27-35 (2021). https://doi.org/10.1007/ s42114-021-00204-5

8. H. Liu, L. Kang, J. Li, F. Liu, X. He et al., Highly efficient deep-blue organic light-emitting diodes based on pyreno 4,5-d imidazole-anthracene structural isomers. J. Mater. Chem. C 7, 10273-10280 (2019). https://doi.org/10.1039/c9tc02990g

9. Z.-Y. Zhang, Y. He, Z. Wang, J. Xu, M. Xie et al., Photochemical phase transitions enable coharvesting of photon energy and ambient heat for energetic molecular solar thermal batteries that upgrade thermal energy. J. Am. Chem. Soc. 142, 12256-12264 (2020). https://doi.org/10.1021/jacs. 0c03748

10. E. Li, Y. Pan, C. Wang, C. Liu, C. Shen et al., Multifunctional and superhydrophobic cellulose composite paper for electromagnetic shielding, hydraulic triboelectric nanogenerator and Joule heating applications. Chem. Eng. J. 420, 129864 (2021). https://doi.org/10.1016/j.cej.2021.129864

11. E. Li, Y. Pan, C. Wang, C. Liu, C. Shen et al., Asymmetry superhydrophobic textiles for electromagnetic interference shielding, photothermal conversion, and solar water evaporation. ACS Appl. Mater. Interfaces 13, 28996-29007 (2021). https://doi.org/10.1021/acsami.1c07976

12. L. Xu, L. Cui, Z. Li, H. Lu, X. Qi et al., Thermodynamic coupling behavior and energy harvesting of vapor grown carbon fiber/graphene oxide/epoxy shape memory composites. Compos. Sci. Technol. 203, 108583 (2021). https://doi.org/ 10.1016/j.compscitech.2020.108583

13. T.K. Das, P. Ghosh, N.C. Das, Preparation, development, outcomes, and application versatility of carbon fiber-based polymer composites: a review. Adv. Compos. Hybrid Mater. 2, 214-233 (2019). https://doi.org/10.1007/s42114-018-0072-Z

14. N. Wen, Z. Fan, S. Yang, Y. Zhao, T. Cong et al., Highly conductive, ultra-flexible and continuously processable PEDOT:PSS fibers with high thermoelectric properties for wearable energy harvesting. Nano Energy 78, 105361 (2020). https://doi.org/10.1016/j.nanoen.2020.105361

15. E.C. Macabutas, A.F. Tongco, Determination of thermal conductivity of bamboo plyboard as thermal insulator for passive roof cooling. Adv. Compos. Hybrid Mater. 4, 647-661 (2020). https://doi.org/10.1007/s42114-020-00185-x

16. H. Kang, H. Kim, S. Kim, H.J. Shin, S. Cheon et al., Mechanically robust silver nanowires network for triboelectric 
nanogenerators. Adv. Funct. Mater. 26, 7717-7724 (2016). https://doi.org/10.1002/adfm.201603199

17. Y. Jia, R. Sun, Y. Pan, X. Wang, Z. Zhai et al., Flexible and thin multifunctional waterborne polyurethane/Ag film for high-efficiency electromagnetic interference shielding, electro-thermal and strain sensing performances. Compos. Part B: Eng. 210, 108668 (2021). https://doi.org/10.1016/j.compo sitesb.2021.108668

18. Y. Feng, X. Jiang, E. Ghafari, B. Kucukgok, C. Zhang et al., Metal oxides for thermoelectric power generation and beyond. Adv. Compos. Hybrid Mater. 1, 114 (2018). https://doi.org/10. 1007/s42114-017-0011-4

19. X. Wang, X. Liu, D.W. Schubert, Highly sensitive ultrathin flexible thermoplastic polyurethane/carbon black fibrous film strain sensor with adjustable scaffold networks. Nano-Micro Lett. 13, 64 (2021). https://doi.org/10.1007/ s40820-021-00592-9

20. H. Cheng, Y. Pan, Q. Chen, R. Che, G. Zheng et al., Ultrathin flexible poly(vinylidene fluoride)/MXene/silver nanowire film with outstanding specific EMI shielding and high heat dissipation. Adv. Compos. Hybrid Mater. 4, 505-513 (2021). https:// doi.org/10.1007/s42114-021-00224-1

21. G. Cai, J.-H. Ciou, Y. Liu, Y. Jiang, P.S. Lee, Leaf-inspired multiresponsive MXene-based actuator for programmable smart devices. Sci. Adv. 5, eaaw7956 (2019). http://doi. org/https://doi.org/10.1126/sciadv.aaw7956

22. C.F. Du, X. Sun, H. Yu, W. Fang, Y. Jing et al., $V_{4} C_{3} T_{x}$ MXene: A promising active substrate for reactive surface modification and the enhanced electrocatalytic oxygen evolution activity. InfoMat 2, 950-959 (2020). https://doi.org/10. 1002/inf2.12078

23. Q. Gao, Y. Pan, G. Zheng, C. Liu, C. Shen et al., Flexible multilayered MXene/thermoplastic polyurethane films with excellent electromagnetic interference shielding, thermal conductivity, and management performances. Adv. Compos. Hybrid Mater. 4, 274-285 (2021). https://doi.org/10.1007/ s42114-021-00221-4

24. W. He, X. Fu, D. Zhang, Q. Zhang, K. Zhuo et al., Recent progress of flexible/wearable self-charging power units based on triboelectric nanogenerators. Nano Energy 84, 105880 (2021). https://doi.org/10.1016/j.nanoen.2021.105880

25. A.A. Mathew, A. Chandrasekhar, S. Vivekanandan, A review on real-time implantable and wearable health monitoring sensors based on triboelectric nanogenerator approach. Nano Energy 80, 150566 (2021). https://doi.org/10.1016/j.nanoen. 2020.105566

26. H. Li, X. Zhang, L. Zhao, D. Jiang, L. Xu et al., A hybrid biofuel and triboelectric nanogenerator for bioenergy harvesting. Nano-Micro Lett. 12, 50 (2020). https://doi.org/10.1007/ s40820-020-0376-8

27. Z.L. Wang, Triboelectric nanogenerators as new energy technology for self-powered systems and as active mechanical and chemical sensors. ACS Nano 7, 9533-9557 (2013). https://doi. org/10.1021/nn404614z

28. Y. Guo, Y. Cao, Z. Chen, R. Li, W. Gong et al., Fluorinated metal-organic framework as bifunctional filler toward highly improving output performance of triboelectric nanogenerators. Nano Energy 70, 104517 (2020). https://doi.org/10.1016/j. nanoen.2020.104517

29. A. Sohn, J.H. Lee, H.-J. Yoon, H.H. Lee, S.W. Kim, Selfboosted power generation of triboelectric nanogenerator with glass transition by friction heat. Nano Energy 74, 104840 (2020). https://doi.org/10.1016/j.nanoen.2020.104840

30. H. Sun, Y. Zhao, C. Wang, K. Zhou, C. Yan et al., UltraStretchable, durable and conductive hydrogel with hybrid double network as high performance strain sensor and stretchable triboelectric nanogenerator. Nano Energy 76, 105035 (2020). https://doi.org/10.1016/j.nanoen.2020.105035

31. H. Jiang, H. Lei, Z. Wen, J. Shi, D. Bao et al., Charge-trapping-blocking layer for enhanced triboelectric nanogenerators. Nano Energy 75, 105011 (2020). https://doi.org/10.1016/j. nanoen.2020.105011

32. K. Tao, Z. Chen, H. Yi, R. Zhang, Q. Shen et al., Hierarchical honeycomb-structured electret/triboelectric nanogenerator for biomechanical and morphing wing energy harvesting. Nano-Micro Lett. 13, 123 (2021). https://doi.org/10.1007/ s40820-021-00644-0

33. M. Zhang, H. Du, K. Liu, S. Nie, T. Xu et al., Fabrication and applications of cellulose-based nanogenerators. Adv. Compos. Hybrid Mater. (2021). https://doi.org/10.1007/ s42114-021-00312-2

34. M.K. Khristosov, S. Dishon, I. Noi, A. Katsman, B. Pokroy, Pore and ligament size control, thermal stability and mechanical properties of nanoporous single crystals of gold. Nanoscale 9, 14458-14466 (2017). https://doi.org/10.1039/c7nr04004k

35. G.-H. Lim, N. Kwon, E. Han, S. Bok, S.-E. Lee et al., Flexible nanoporous silver membranes with unprecedented high effectiveness for electromagnetic interference shielding. J. Ind. Eng. Chem. 93, 245-252 (2021). https://doi.org/10.1016/j.jiec.2020.09.030

36. T.H. Park, S. Yu, M. Koo, H. Kim, E.H. Kim et al., Shapeadaptable 2D titanium carbide (MXene) heater. ACS Nano 13, 6835-6844 (2019). https://doi.org/10.1021/acsnano.9b01602

37. B. Zhou, M. Su, D. Yang, G. Han, Y. Feng et al., Flexible MXene/silver nanowire-based transparent conductive film with electromagnetic interference shielding and electrophoto-thermal performance. ACS Appl. Mater. Interfaces 12, 40859-40869 (2020). https://doi.org/10.1021/acsami.0c09020

38. S. Kimura, T. Sugita, K. Nakamura, N. Kobayashi, An improvement in the coloration properties of Ag depositionbased plasmonic EC devices by precise control of shape and density of deposited Ag nanoparticles. Nanoscale 12, 2397523983 (2020). https://doi.org/10.1039/d0nr05196a

39. A. Loiseau, L. Zhang, D. Hu, M. Salmain, Y. Mazouzi et al., Core-Shell gold/silver nanoparticles for localized surface plasmon resonance-based naked-eye toxin biosensing. ACS Appl. Mater. Interfaces 11, 46462-46471 (2019). https://doi.org/10. 1021/acsami.9b14980

40. R. Li, L. Zhang, L. Shi, P. Wang, MXene $\mathrm{Ti}_{3} \mathrm{C}_{2}$ : An effective 2D light-to-heat conversion material. ACS Nano 11, 37523759 (2017). https://doi.org/10.1021/acsnano.6b08415

41. H. Riazi, S.K. Nemani, M.C. Grady, B. Anasori, M. Soroush, $\mathrm{Ti}_{3} \mathrm{C}_{2}$ MXene-polymer nanocomposites and their applications. 
J. Mater. Chem. A 9, 8051-8098 (2021). https://doi.org/10. 1039/d0ta08023c

42. A. Mohammadi, M. Barikani, A.H. Doctorsafaei, A.P. Isfahani, E. Shams et al., Aqueous dispersion of polyurethane nanocomposites based on calix 4 arenes modified graphene oxide nanosheets: Preparation, characterization, and anti-corrosion properties. Chem. Eng. J. 349, 466-480 (2018). https:// doi.org/10.1016/j.cej.2018.05.111

43. V. Slabov, S. Kopyl, M.P. Soares dos Santos, A.L. Kholkin, Natural and eco-friendly materials for triboelectrie energy harvesting. Nano-Micro Lett. 12, 42 (2020). https://doi.org/ 10.1007/s40820-020-0373-y

44. K. Zhao, Y. Wang, L. Han, Y. Wang, X. Luo et al., Nanogenerator-based self-charging energy storage devices. Nano-Micro Lett. 11, 19 (2019). https://doi.org/10.1007/ s40820-019-0251-7

45. K. Zhou, Y. Zhao, X. Sun, Z. Yuan, G. Zheng et al., Ultrastretchable triboelectric nanogenerator as high-sensitive and self-powered electronic skins for energy harvesting and tactile sensing. Nano Energy 70, 104546 (2020). https://doi.org/10. 1016/j.nanoen.2020.104546

46. C. Ye, S. Dong, J. Ren, S. Ling, Ultrastable and high-performance silk energy harvesting textiles. Nano-Micro Lett. 12, 12 (2020). https://doi.org/10.1007/s40820-019-0348-Z

47. H. Sun, Y. Zhao, S. Jiao, C. Wang, Y. Jia et al., Environment tolerant conductive nanocomposite organohydrogels as flexible strain sensors and power sources for sustainable electronics. Adv. Funct. Mater. 31, 2101696 (2021). https:// doi.org/10.1002/adfm.202101696

48. Y. Zhang, Q. Zeng, Y. Wu, J. Wu, S. Yuan et al., An ultra-durable windmill-like hybrid nanogenerator for steady and efficient harvesting of low-speed wind Eenergy. Nano-Micro Lett. 12, 175 (2020). https://doi.org/10.1007/s40820-020-00513-2

49. F. Peng, D. Liu, W. Zhao, G. Zheng, Y. Ji et al., Facile fabrication of triboelectric nanogenerator based on low-cost thermoplastic polymeric fabrics for large-area energy harvesting and self-powered sensing. Nano Energy 65, 104068 (2019). https:// doi.org/10.1016/j.nanoen.2019.104068

50. J. Luo, W. Gao, Z.L. Wang, The triboelectric nanogenerator as an innovative technology toward intelligent sports. Adv. Mater. 33, 2004178 (2021). https://doi.org/10.1002/adma.202004178

51. J. Yi, K. Dong, S. Shen, Y. Jiang, X. Peng et al., Fully fabricbased triboelectric nanogenerators as self-powered humanmachine interactive keyboards. Nano-Micro Lett. 13, 103 (2021). https://doi.org/10.1007/s40820-021-00621-7

52. Z. Hui, M. Xiao, D. Shen, J. Feng, P. Peng et al., A self-powered nanogenerator for the electrical protection of integrated circuits from trace amounts of liquid. Nano-Micro Lett. 12, 5 (2020). https://doi.org/10.1007/s40820-019-0338-1

53. D. Wang, D. Zhang, P. Li, Z. Yang, Q. Mi et al., Electrospinning of flexible Poly (vinyl alcohol)/MXene nanofiber-based humidity sensor self-powered by monolayer molybdenum diselenide piezoelectric nanogenerator. Nano-Micro Lett. 13, 57 (2021). https://doi.org/10.1007/s40820-020-00580-5 